\title{
Sechs-Minuten-Gehtest mit und ohne Sauerstoff bei Patienten mit COPD: Vergleich von Gehstrecke und Sauerstoffsättigung bei unterschiedlichen Applikationsformen
}

\author{
N. Weber ${ }^{1}$ \\ P. Brand ${ }^{2}$ \\ M. Kohlhäufl' \\ K. Häußinger ${ }^{1}$
}

\author{
Six-Minute-Walking-Test with and without Oxygen in Patients with COPD: \\ Comparison of Walking Distance and Oxygen Saturation in Varying Forms \\ of Application
}

\section{Zusammenfassung}

Hintergrund: Patienten mit COPD und respiratorischer Insuffizienz erfahren unter Belastung meist einen Abfall der Sauerstoffsättigung $\left(\mathrm{SaO}_{2}\right)$. Fragestellung unserer Untersuchung war, wie sich im Sechs-Minuten-Gehtest (6MWT) Gehstrecke und $\mathrm{SaO}_{2}$ unter Sauerstoff $\left(\mathrm{O}_{2}\right) 2 \mathrm{l} / \mathrm{min}$ kontinuierlichem Fluss (CF), Demand (D) und Raumluft (RL) ändern und wie sich die 10 Patienten mit kürzester (Gruppe I) gegenüber den 10 Patienten mit längster (Gruppe II) Gehstrecke im Ausgangs-6MWT (unter CF) unterscheiden. Methode und Patienten: Bei 27 Patienten wurde an 3 folgenden Tagen ein 6MWT mit CF, D und RL durchgeführt. Über 6 Min vor (Phase 1), während (Phase 2) und nach (Phase 3) dem 6MWT wurden $\mathrm{O}_{2} \mathrm{CF}$ (Tag 1) bzw. D (Tag 2) bzw. RL (Tag 3) verabfolgt, die durchschnittliche $\mathrm{SaO}_{2}$ jeder Phase gemessen und ihre Änderung $\left(\Delta \mathrm{SaO}_{2}\right)$ im und nach dem 6MWT errechnet. Zudem wurde die $\Delta \mathrm{SaO}_{2}$ zwischen Beginn und Mitte sowie zwischen Mitte und Ende jeder Phase gemessen. Zeitnah zu den Untersuchungen wurden $\mathrm{FEV}_{1}$ und $\mathrm{pO}_{2}$ bestimmt. Ergebnisse: Im Gesamtkollektiv änderte sich die Gehstrecke unter den 3 Applikationsformen nicht signifikant. Unter allen Applikationsformen trat ein hoch signifikanter Abfall der $\mathrm{SaO}_{2}$ ein. Die Abnahme unter den beiden Formen der $\mathrm{O}_{2}$-Applikation unterschied sich nicht signifikant. Der Abfall der $\mathrm{SaO}_{2}$ unter Belastung und der nachfolgende Wiederanstieg in Ruhe traten in den ersten 3 Minuten der

\section{Abstract}

Background: Patients afflicted with COPD and respiratory Failure often experience a decrease of oxygen saturation $\left(\mathrm{SaO}_{2}\right)$ under physical strain. The aim of our study was to find out which changes occur in walking distance and $\mathrm{SaO}_{2}$ under the application of Oxygen $\left(\mathrm{O}_{2}\right) 2 \mathrm{l} / \mathrm{min}$ continous flow, under demand (D) and under normal air (RL) when performing the Six-MinuteWalking-Test (6MWT) and further, in which way do the 10 patients of the shortest walking distance group (group I) differ from the eo patients with the longest walking distance (group II) concerning the starter 6 MWT (under CF). Method and Patients: 27 patients undertook a 6MWT three days in a row with CF, D and (RL), 6 minutes before (phase I), during (phase II) and after (phase III) the 6MWT oxygen was applied in CF (day I), $\mathrm{D}$ (day II) and RL (day III), the mean oxygen saturation in each phase was measured and the change $\left(\Delta \mathrm{SaO}_{2}\right)$ during and after the 6MWT was calculated. Additionally we measured the $\Delta \mathrm{SaO}_{2}$ between start and middle, as well as between middle and end of each phase. In close proximity to the tests $\mathrm{FEV}_{1}$ and $\mathrm{pO}_{2}$ was determined. Results: In the total of all test persons there was no significant difference in the walking distance tetween the 3 forms of application. There was, however, a highly significant decrease of the oxygen saturation. The decrease in the two forms of oxygen application did not differ significantly. The decrease of

Institutsangaben

${ }^{1}$ Asklepios Fachkliniken München-Gauting, Klinik für Pneumologie und Thoraxchirurgie

(Leiter: Prof. Dr. K. Häußinger)

${ }^{2}$ Inamed $\mathrm{GmbH}$, Gauting

Anmerkung

Teile des Inhalts wurden als Vortrag beim 46. Kongress der Deutschen Gesellschaft für Pneumologie,

März 2005, Berlin unter dem Titel „Sauerstoffsättigungsprofil bei Patienten mit COPD

und respiratorischer Insuffizienz im Sechs-Minuten-Gehtest“ vorgetragen.

Korrespondenzadresse

Dr. med. Norbert Weber · Asklepios Fachkliniken München-Gauting, · Klinik für Pneumologie und Thoraxchirurgie (Leiter: Prof. Dr. K. Häußinger) · Robert Koch-Allee 2 · 82131 Gauting ·

E-mail: n.weber@asklepios.com

Eingang: 10. Februrar 2005 • Nach Revision alkzeptiert: 20. Oktober 2005

Bibliografie

Pneumologie 2006; 60: 220-228 @ Georg Thieme Verlag KG Stuttgart · New York

DOI $10.1055 / \mathrm{s}-2005-919094$

ISSN 0934-8387 
jeweiligen Phase auf. Patienten der Gruppe II konnten die Gehstrecke unter RL häufig weiter steigern, bei den Patienten der Gruppe I nahm jedoch die Gehstrecke weiter ab. In Gruppe II war das $\mathrm{FEV}_{1}$ abs. signifikant höher, $\mathrm{FEV}_{1} \%$ Soll und $\mathrm{pO}_{2}$ hingegen waren zwischen beiden Gruppen nicht signifikant different. Schlussfolgerung: Patienten mit langer Gehstrecke zeigen selbst unter RL einen deutlichen Lerneffekt, haben ein höheres $\mathrm{FEV}_{1}$, nicht jedoch einen höheren $\mathrm{pO}_{2}$. Die Phase der Wiederaufsättigung nach Belastungsende ist kurz. Sauerstoffzufuhr über ein Demand-Ventil ist bei einer Flussrate von $21 /$ min einem kontinuierlichen Fluss nicht unterlegen. In mehr als der Hälfte der Patienten fällt bei beiden $\mathrm{O}_{2}$-Applikationsformen jedoch die $\mathrm{SaO}_{2}$ auf Werte unter $90 \%$ ab, so dass in diesen Fällen die Flussrate zu erhöhen ist.
$\mathrm{SaO}_{2}$ under strain and the consecutive rise under rest occurred within the first 3 minutes of each phase. Patients of group II were often able to increase their walking distance under RL, whereas patients of group I decreased their walking distance further. In group II $\mathrm{FEV}_{1}$ abs. was significantly higher, but $\mathrm{FEV}_{1} \%$ debit and $\mathrm{pO}_{2}$ did not differ significantly between both groups. Conclusions: Patients with a long walking distance showed a marked learning effect even under RL, they presented a higher $\mathrm{FEV}_{1}$, but not an increased $\mathrm{pO}_{2}$. The phase of resaturation after the end of strain is short. Oxygen application through a demand valve with a flow rate of $2 \mathrm{l} / \mathrm{min}$ is not inferior to a continuous flow. In more than half of all patients the $\mathrm{SaO}_{2}$ fell below $90 \%$ under both forms of oxygen application, in conclusion the flow rate should be increased in these cases.

\section{Einleitung}

Häufigste Leitsymptome von Patienten mit chronisch obstruktiver Lungenerkrankung (COPD) sind eingeschränkte Belastbarkeit bzw. Atemnot bei Anstrengung. Patienten mit chronischer Hypoxämie (Stadium IV nach GOLD) weisen eine verminderte Lebensqualität und Leistungsfähigkeit auf [1,2]; Morbidität und Mortalität sind erhöht [3]. Eine Langzeit-Sauerstofftherapie verlängert die Lebenserwartung von Patienten mit einem Sauerstoffpartialdruck $\left(\mathrm{pO}_{2}\right) \leq 55$ Torr $[4,5]$. Ziele und Indikationen sind in den „Leitlinien zur Langzeit-Sauerstofftherapie“ angeführt [6]. Viele Patienten mit COPD haben einen Sauerstoffpartialdruck von $>55$ Torr in Ruhe, zeigen jedoch eine signifikante Hypoxämie unter Belastung [7,8]. Mit tragbaren Sauerstoffgeräten ausgestattete Patienten haben eine höhere Lebensqualität [9]. Sauerstoffzufuhr unter Belastung soll das Atemnotempfinden und die eintretende Abnahme der Sauerstoffsättigung $\left(\mathrm{SaO}_{2}\right)$ reduzieren $[10,11]$ sowie die Belastbarkeit verbessern $[12,13]$, selbst bei COPD-Patienten ohne Hypoxämie [14]. Andere Studien konnten diese Ergebnisse nicht bestätigen [15,16]. O'Donnell wies einen Anstieg der Belastungsdauer, eine Abnahme der Ventilation und der Ermüdung der Beine nach, verabreichte aber 60\% Sauerstoff [17]. Vergleichbare Ergebnisse bot eine Untersuchung von Chatila: Im Vergleich zu einem Niedrig-Fluss-Sauerstoff-System führte befeuchteter und angewärmter Hoch-FlussSauerstoff bei gleicher Fraktion des eingeatmeten Sauerstoffs $\left(\mathrm{FIO}_{2}\right)$ zu einer Verlängerung der Belastungszeit bei geringerer Atemnot und Abnahme von Atemfrequenz, des Quotienten aus Atemfrequenz und Atemzugvolumen sowie zu einer Abnahme der Inspirationszeit im Verhältnis zur Atemzugsdauer [18]. Der Einfluss auf Ausdauer und Atemnot ist dosisabhängig. Neben der Reduktion von Atemfrequenz und Laktatproduktion ist auch die Abnahme der Überblähung unter Sauerstoff eine Erklärung für den günstigen Effekt [19].

Als Belastungsform wird häufig der Sechs-Minuten-Gehtest (6MWT) herangezogen. Er ist ohne größeren Aufwand an Personal oder spezieller technischer Ausstattung durchzuführen und ist für die Patienten angenehmer als andere Belastungsuntersuchungen $[20,21]$. Der 6MWT stellt bei Patienten mit COPD ein Maß für die Belastbarkeit im Alltag und den funktionellen Status dar $[22,23]$. Er eignet sich ebenso als Maß für den Erfolg der Rehabilitation [22]. Eine Reihe von Faktoren außerhalb des Atem- systems $[23,24]$ wie Geschlecht, Alter, Größe und Gewicht, beeinflusst die Gehstrecke, so dass diese auch in Prozent des Sollwertes angegeben werden sollte $[25,26]$. Bei intraindividuellen Untersuchungen ist dies jedoch ohne Belang. Die Überlebenszeit von Patienten mit COPD steht mit der Gehstrecke in statistischem Zusammenhang [24,27]. Sie stellt einen besseren Prädiktor der Mortalität dar als das Forcierte Exspiratorische Volumen $\left(\mathrm{FEV}_{1}\right)[28]$.

Um die Wertigkeit des 6MWT unter verschiedenen Bedingungen zu analysieren, haben wir folgende Fragen untersucht:

- Wie lange ist, bzw. in welchem Ausmaß ändert sich die Gehstrecke im 6MWT

- unter einer Sauerstoffinsufflationsrate von $21 / \mathrm{min}$

- mit kontinuierlichem Fluss (CF)

- mit einem Demand-System(D)

- unter Raumluft (RL)?

- In welchem Ausmaß korrelieren die Gehstrecken unter CF und D sowie unter CF und RL im Gesamtkollektiv? Lässt sich (in einer Subgruppe) ein Lerneffekt erkennen?

- Bei welchen Parametern unterscheidet sich die Gruppe der 10 Patienten mit der kürzesten von der Gruppe der 10 Patienten mit der längsten Gehstrecke unter CF?

- In welchem Ausmaß ändert sich die durchschnittliche (über eine Phase von jeweils 6 Minuten gemittelte) $\mathrm{SaO}_{2}$ während und nach Belastung? Ergeben sich Unterschiede zwischen den drei Applikationsformen? Ist die Änderung der $\mathrm{SaO}_{2}$ in den jeweils 6 Minuten vor, während und nach Belastung mit und ohne Sauerstoffzufuhr linear oder weist sie einen Kurvenverlauf auf?

\section{Patienten und Methoden}

Untersucht wurden 27 Patienten mit COPD und unterschiedlich ausgeprägter respiratorischer Insuffizienz in einer stabilen Phase. Die Patienten wurden über den Ablauf der Untersuchung aufgeklärt und dokumentierten durch Unterschrift ihre Bereitschaft zur Teilnahme. Sie wurden gebeten, mindestens 15 Minuten vor Untersuchungsbeginn eine eventuell vorausgehende Sauerstoffzufuhr zu unterbrechen und ein kurz wirksames Beta ${ }_{2}$-Adrenergikum zu inhalieren. Anschließend wurden unmittelbar nach Beginn einer Ruhephase von 6 Minuten $\mathrm{O}_{2} 2 \mathrm{l} / \mathrm{min} \mathrm{CF}$ über ein por- 


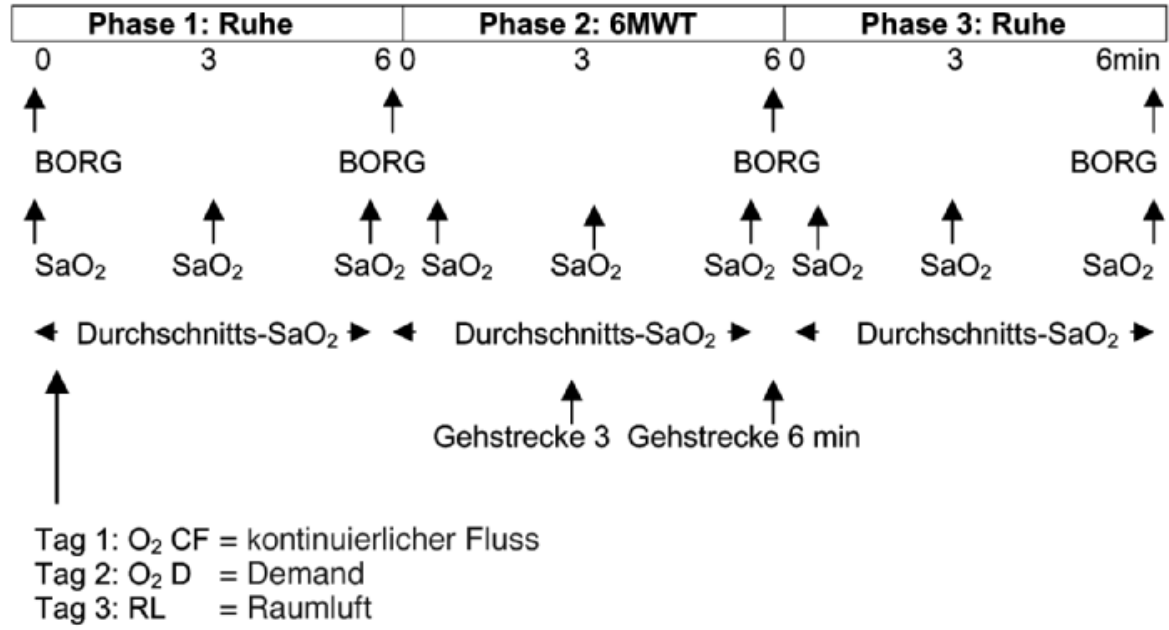

tables Flüssig-Sauerstoffsystem (SPIRIT 300 ${ }^{\circledR}$, Füllgewicht $1,95 \mathrm{~kg}$ ) verabfolgt (Phase 1). Es folgte der 6MWT in einem Korridor von 25 m Länge (Phase 2). Nach Belastungsende schloss sich eine weitere Ruhephase von 6 Minuten an (Phase 3). In jeder der drei Phasen wurden mittels eines Pulsoximeters (SpiroPro®, Fa. JAEGER) $\mathrm{SaO}_{2}$ und Puls kontinuierlich gemessen und der jeweilige Durchschnittswert ermittelt. Festgehalten wurde auch die $\mathrm{SaO}_{2}$ zu Beginn, nach 3 und nach 6 Minuten der jeweiligen Phase. Zu Untersuchungsbeginn und am Ende jeder Phase wurden die Patienten gebeten, den Schweregrad ihrer gegenwärtigen Atemnot anhand der BORG-Skala zu bestimmen [29]. Die Gehstrecke wurde nach 3 und nach 6 Minuten dokumentiert, die Strecke in den ersten 3 Minuten in Prozent der Gesamtgehstrecke ermittelt. Der Untersuchungsablauf ist in Abb. 1 dargestellt. An zwei weiteren Tagen wurde der Versuch mit $\mathrm{O}_{2} 2 \mathrm{l} / \mathrm{min} \mathrm{D}$, dann unter Raumluft, stets in dieser Reihenfolge, wiederholt. Der gleiche Untersucher begleitete alle 27 Patienten bei allen Untersuchungen, um einen Untersucher-Bias zu vermeiden. Der Abstand zwischen zwei Untersuchungen betrug in der Regel 24, in wenigen Ausnahmen 48 bzw. 72 Stunden. Die bronchospasmolytische (kurzund langwirksame Beta-2-Adrenergika und Anticholinergika, Theophyllin) und antientzündliche (inhalative und/oder systemische Corticoide) Behandlung wurde während des Untersuchungszeitraums weitestgehend konstant gehalten. Um Ermüdungserscheinungen durch wiederholte Belastung auszuschließen wurden die Untersuchungen nicht am gleichen Tag vorgenommen. Sie wurden weitgehend zur gleichen Tageszeit durchgeführt, um tageszeitliche Leistungsschwankungen zu minimieren, die als Folge der im Tagesverlauf abnehmenden Wirkung der lang wirksamen Bronchospasmolytika auftreten können. Die Patienten trugen bei allen drei Applikationsformen das gefüllte Flüssig-Sauerstoffsystem mit sich, nahmen jedoch am Tag 3 keinen Sauerstoff. Zeitnah zu den drei Untersuchungen wurden Sauerstoff- und Kohlendioxidpartialdruck $\left(\mathrm{pO}_{2}, \mathrm{pCO}_{2}\right)$ sowie $\mathrm{FEV}_{1}$ absolut und in Prozent des altersentsprechenden Sollwerts $\left(\mathrm{FEV}_{1} \%\right.$ Soll) ermittelt. Ferner wurden die Patienten gebeten, anhand der MRC (Medical Research Council)-Skala ihr DyspnoeEmpfinden im Alltag anzugeben [30]. Die Sollwerte für den $6 \mathrm{MWT}$ wurden aus den geschlechtsspezifischen Regressionsgleichungen nach Enright errechnet [25].
Abb. 1 Untersuchungsablauf.

\section{Statistik}

Gruppenunterschiede wurden unter Verwendung des WilcoxonMan-Whitney-Test auf Signifikanzen getestet. Irrtumswahrscheinlichkeiten mit einem $\mathrm{p}$-Wert $<0,05$ wurden als statistisch signifikant gewertet.

\section{Ergebnisse}

Die Charakteristika der untersuchten Patienten sind aus Tab. 1 zu entnehmen.

Tab. 2 zeigt die Durchschnittswerte der Gehstrecke des Gesamtkollektivs. Sie nimmt unter $\mathrm{O}_{2} 2 \mathrm{l} / \mathrm{min} \mathrm{D}$ gegenüber $\mathrm{O}_{2} 2 \mathrm{l} / \mathrm{min} \mathrm{CF}$ um $6,2 \%$ zu. Unter RL liegt die Zunahme gegenüber CF bei $4,6 \%$. Die Gehstrecken unterscheiden sich unter den 3 Applikationsformen nicht signifikant.

In Abb. 2 und $\mathbf{3}$ sind die Korrelationen $\mathrm{r}$ der Gehstrecken zwischen CF und D sowie zwischen CF und RL dargestellt. Die Regressionsgerade ist durch die Formel $y=a x+b \quad(a=$ Steigung, $\mathrm{b}=$ Schnittpunkt mit der $\mathrm{y}$-Achse) beschrieben. Geht man von der Annahme aus, dass die Applikationsformen unter CF und D äquivalent sind, ergibt sich eine enge Korrelation zwischen den

Tab. 1 Beschreibung des Patientenkollektivs

\begin{tabular}{|ll}
\hline Geschlecht & $\begin{array}{c}\text { Männer: } \mathbf{1 2} \\
\text { Frauen: } \mathbf{1 5}\end{array}$ \\
\hline Durchschnittsalter & $66,0 \pm 8,9$ J \\
\hline Body-Mass-Index (BMI) & $26,3 \pm 5,6 \mathrm{~kg} / \mathrm{m} 2$ \\
\hline durchschnittlicher $\mathrm{pO}_{2}$ & $55,4 \pm 7,3 \mathrm{Torr}$ \\
\hline durchschnittlicher $\mathrm{PCO}_{2}$ & $40,5 \pm 6,4 \mathrm{Torr}$ \\
\hline durchschnittliche FEV1 & $1,06 \pm 0,45$ I \\
\hline durchschnittliche FEV1\% Soll & $44,2 \pm 17,5 \%$ \\
\hline durchschnittlicher MRC-Wert & $2,8 \pm 0,9$ \\
\hline durchschnittliche Soll-Gehstrecke & $500 \pm 65 \mathrm{~m}$ \\
n. Enright & $(54,7 \pm 13,6 \%$ Soll) \\
\hline
\end{tabular}


Tab. 2 Gehstrecke, durchschnittliche $\mathrm{SaO}_{2}$ vor, unter und nach Belastung sowie Änderung der $\mathrm{SaO}_{2}(\Delta \mathrm{SaO})$ unter $\mathrm{Belastung} ; \Delta \mathrm{SaO}$ in den ersten und zweiten drei min der jeweiligen Phase vor, unter und nach Belastung; $\triangle B O R G$ : Änderung BORG nach/vor 6MWT

\begin{tabular}{|c|c|c|c|c|}
\hline Messwert & $\begin{array}{l}\mathrm{O}_{2} \\
\text { kontinuierlicher Fluss } \\
\text { (CL) }\end{array}$ & $\begin{array}{l}\mathrm{O}_{2} \\
\text { Demand } \\
\text { (D) }\end{array}$ & $\begin{array}{l}\text { Raumluft } \\
\text { (RL) }\end{array}$ & $\begin{array}{l}\text { Signifikanz p } \\
\text { a) CF vs. D } \\
\text { b) CF vs. } R L\end{array}$ \\
\hline $\begin{array}{l}\text { Gehstrecke 6MWT (m) } \\
\text { Gehstrecke \% Soll }\end{array}$ & $\begin{array}{c}272 \pm 72 \\
54,7 \pm 13,6\end{array}$ & $\begin{array}{c}289 \pm 83 \\
57,8 \pm 16,1\end{array}$ & $\begin{array}{c}284 \pm 99 \\
57,1 \pm 19,7\end{array}$ & $\begin{array}{l}\text { a) } 0,41 \\
\text { b) } 0,68\end{array}$ \\
\hline $\begin{array}{l}\text { Gehstrecke } \\
\text { 1. } 3 \text { Min (\%) }\end{array}$ & $51,8 \pm 4,1$ & $52,4 \pm 3,4$ & $53,2 \pm 4,5$ & $\begin{array}{l}\text { a) } 0,12 \\
\text { b) } 0,08\end{array}$ \\
\hline $\mathrm{SaO}_{2}$ vor Bel. (\%) (Phase 1) & $93,7 \pm 2,5$ & $93,3 \pm 2,7$ & $90,4 \pm 2,9$ & $\begin{array}{l}\text { a) } 0,63 \\
\text { b) }<0,001\end{array}$ \\
\hline $\mathrm{SaO}_{2}$ unter Bel. (\%) (Phase 2) & $87,9 \pm 6,8$ & $88,2 \pm 5,7$ & $83,2 \pm 5,8$ & $\begin{array}{l}\text { a) } 0,95 \\
\text { b) } 0,12\end{array}$ \\
\hline $\begin{array}{l}\mathrm{SaO}_{2} \text { nach Bel. (\%) } \\
\text { (Phase 3) }\end{array}$ & $94,1 \pm 3,3$ & $94,2 \pm 3,2$ & $90,7 \pm 3,9$ & $\begin{array}{l}\text { a) } 0,85 \\
\text { b) }<0,001\end{array}$ \\
\hline$\triangle \mathrm{SaO}_{2}$ unter Bel. (\%) & $-5,8$ & $-5,2$ & $-7,3$ & $\begin{array}{l}\text { a) } 0,85 \\
\text { b) } 0,16\end{array}$ \\
\hline Signifikanz p der 916; $\mathrm{SaO}_{2}$ & 0,0002 & 0,0002 & $<0,0001$ & \\
\hline$\triangle B O R G$ unter Bel. & $\begin{array}{l}3,2 \\
(2,8 \rightarrow 6,0)\end{array}$ & $\begin{array}{l}3,0 \\
(2,8 \rightarrow 5,8)\end{array}$ & $\begin{array}{l}3,2 \\
(3,0 \rightarrow 6,2)\end{array}$ & $\begin{array}{l}\text { a) } 0,66 \\
\text { b) } 0,94\end{array}$ \\
\hline$\triangle B O R G$ nach Bel. & $\begin{array}{l}-2,6 \\
(6,0 \rightarrow 3,4)\end{array}$ & $\begin{array}{l}-2,7 \\
(5,9 \rightarrow 3,2)\end{array}$ & $\begin{array}{l}-3,2 \\
(6,2 \rightarrow 3,0)\end{array}$ & \\
\hline $\begin{array}{l}\triangle \mathrm{SaO}_{2} \\
\text { 1. } 3 \mathrm{Min} . \text { vor Bel. (\%) } \\
\text { 2. } 3 \mathrm{Min} . \text { vor Bel. (\%) }\end{array}$ & $\begin{array}{l}4,3 \pm 3,0 \\
0,3 \pm 1,0\end{array}$ & $\begin{array}{l}4,0 \pm 2,5 \\
0,6 \pm 1,2\end{array}$ & $\begin{array}{l}-0,3 \pm 1,3 \\
-0,1 \pm 1,4\end{array}$ & $\begin{array}{l}\text { a) } 0,81 \\
\text { b) }<0,001\end{array}$ \\
\hline $\begin{array}{l}\triangle \mathrm{SaO}_{2} \\
\text { 1. } 3 \mathrm{Min} \text {. unter Bel. (\%) } \\
\text { 2. } 3 \mathrm{Min} \text {. unter Bel. (\%) }\end{array}$ & $\begin{array}{r}-7,6 \pm 5,5 \\
0,2 \pm 2,4\end{array}$ & $\begin{array}{l}-6,4 \pm 5,1 \\
-0,2 \pm 2,4\end{array}$ & $\begin{array}{r}-7,8 \pm 5,3 \\
0,1 \pm 1,8\end{array}$ & $\begin{array}{l}\text { a) } 0,50 \\
\text { b) } 0,87\end{array}$ \\
\hline $\begin{array}{l}\triangle \mathrm{SaO}_{2} \\
\text { 1. } 3 \mathrm{Min} \text {. nach Bel. (\%) } \\
\text { 2. } 3 \mathrm{Min} \text {. nach Bel. (\%) }\end{array}$ & $\begin{array}{l}6,3 \pm 6,9 \\
0,0 \pm 1,0\end{array}$ & $\begin{array}{l}5,7 \pm 6,0 \\
0,1 \pm 0,9\end{array}$ & $\begin{array}{l}7,2 \pm 6,1 \\
0,0 \pm 1,2\end{array}$ & $\begin{array}{l}\text { a) } 0,87 \\
\text { b) } 0,41\end{array}$ \\
\hline
\end{tabular}

\section{Korrelation CF / D}

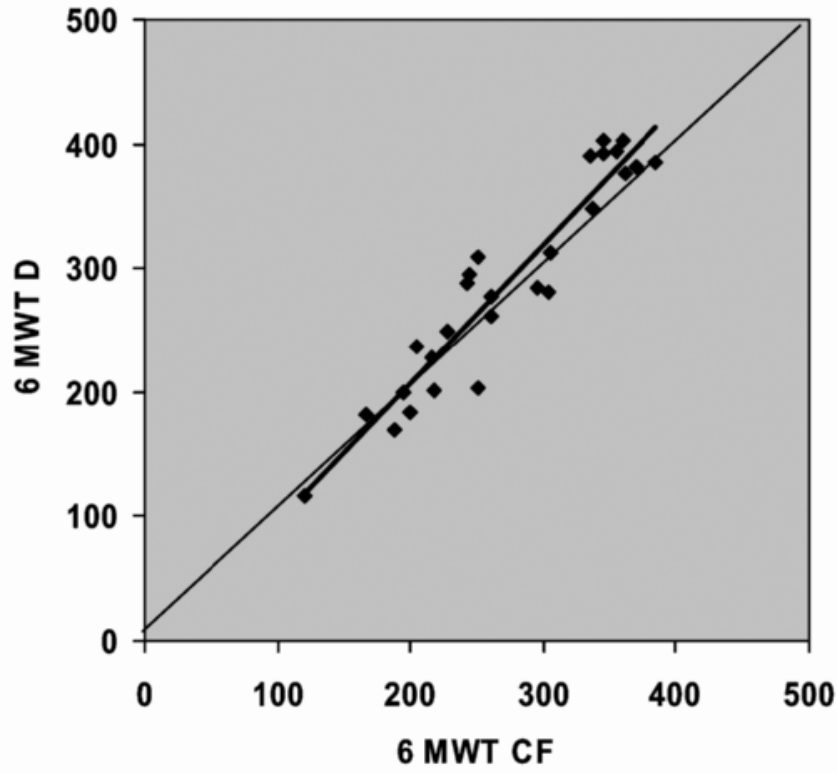

Abb. 2 Korrelation zwischen Gehstrecke unter kontinuierlichem Fluss (CF) und Demand (D) $(r=0,95, p<0,0001)$.

Gehstrecken der einzelnen Patienten $(r=0,95, p<0,0001$; Abb. 2). Die Steigung a der Regressionsgeraden entspricht mit 1,12 nahezu der sog. „line of identity“. Hieraus lässt sich ein hoher Grad an Reproduzierbarkeit ableiten. Vergleicht man hingegen die Gehstrecke unter CF mit der Gehstrecke unter RL
Korrelation CF / RL

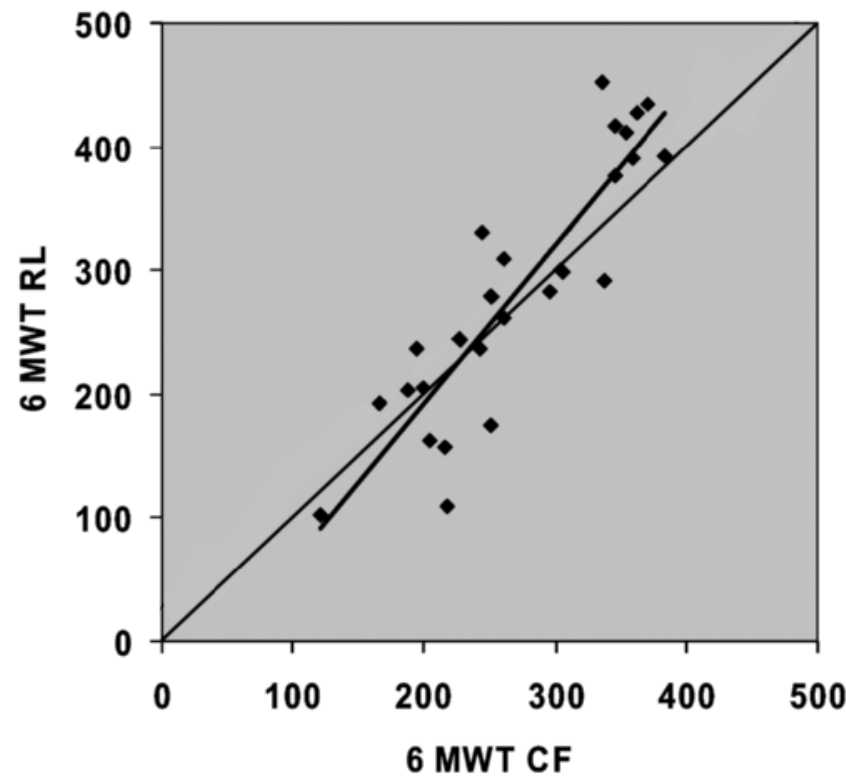

Abb. 3 Korrelation zwischen Gehstrecke unter kontinuierlichem Fluss (CF) und Raumluft (RL) $(r=0,89, p<0,0001)$.

(Abb. 3), ist die Korrelation mit $r=0,89$ geringer, die Steigung a mit 1,28 höher als in Abb. 2. Bei Annahme, dass unter RL die Belastungsbedingungen ungünstiger sind als unter $\mathrm{CF}$, stellt sich der Lerneffekt in einem Subkollektiv deutlich dar: aus der höheren Steigung von 1,28 ist abzuleiten, dass bei Patienten mit kur- 


\section{Gehstrecke 6MWT [m] Gr. I u. Gr. II}

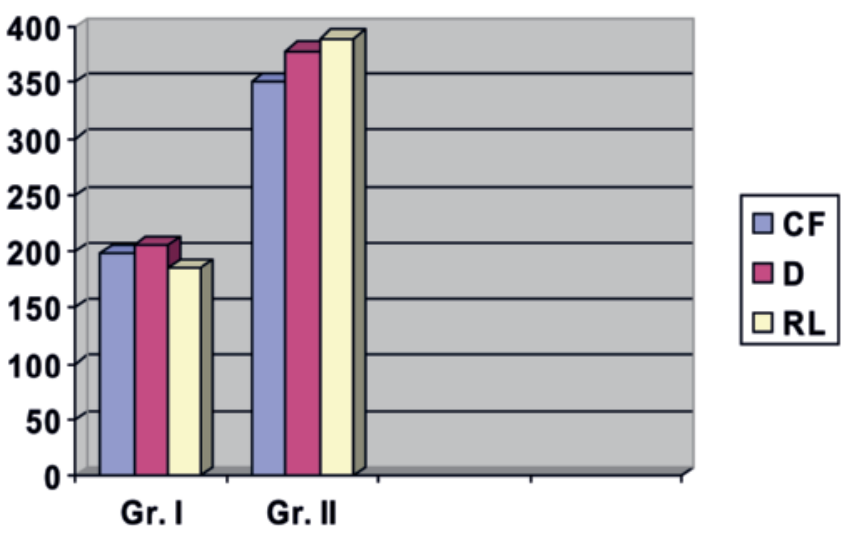

Abb. 4 Gehstrecke unter $2 \mathrm{I} \mathrm{O}_{2}$ kontinuierlichem Fluss (CF), $2 \mathrm{I} \mathrm{O}_{2}$ Demand (D) und unter Raumluft (RL) in der Gruppe mit kürzester (I) und längster (II) Ausgangsgehstrecke.

zer Gehstrecke unter CF die Gehstrecke unter RL abnimmt, im Subkollektiv mit langer Gehstrecke jedoch weiter ansteigt. Werden die 10 Patienten mit der kürzesten (Gruppe I, Gehstrecke $198 \pm 34 \mathrm{~m}$ ) den 10 Patienten mit der längsten Gehstrecke (Gruppe II, Gehstrecke $350 \pm 22 \mathrm{~m}$ ) gegenübergestellt (Abb.4, Tab 3), ergibt sich in Bezug auf die Änderung der Gehstrecke unter D

\section{$\Delta \mathrm{SaO}_{2}[\%]$}

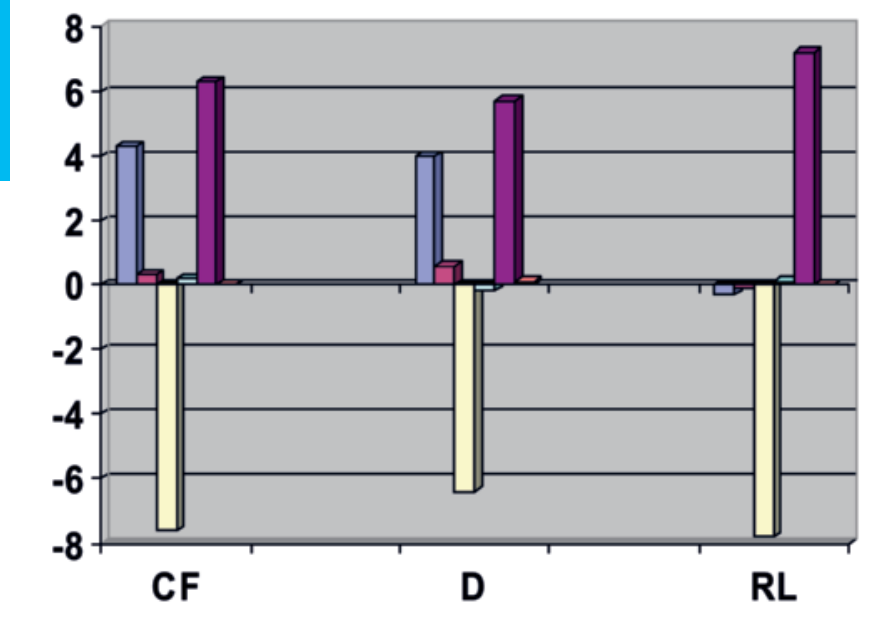

noch kein signifikanter Unterschied (in Gruppe I Zunahme um $8 \pm 22 \mathrm{~m}$, in Gruppe II Zunahme um $29 \pm 21 \mathrm{~m}, \mathrm{p}=0,08$ ); unter RL jedoch ist die Differenz (bei Abnahme in Gruppe I um $13 \pm$ $45 \mathrm{~m}$ und Zunahme in Gruppe II um $40 \pm 46 \mathrm{~m}$ ) signifikant $(p=0,02)$. Charakterisiert ist diese Gruppe durch ein signifikant höheres absolutes $\mathrm{FEV}_{1}$, obgleich sich kein signifikanter Unterschied in Bezug auf das $\mathrm{FEV}_{1} \%$ Soll oder den $\mathrm{pO}_{2}$ ergibt. Die Abnahme der $\mathrm{SaO}_{2}$ ist in Gruppe II geringer ausgeprägt, jedoch nicht signifikant.

Trotz einer Sauerstoffzufuhr von 2 1/min tritt unter CF bei 16, unter $\mathrm{D}$ bei 14 Patienten ein Abfall der durchschnittlichen $\mathrm{SaO}_{2}$ auf Werte $<90 \%$ (unter CF Abfall bis maximal 22,5, unter D bis 15,7 Prozentpunkte) ein. Unter RL ist bei 23 Patienten eine Abnahme $<90 \%$ (bis maximal 18,5 Prozentpunkte) zu verzeichnen. Die Abnahme der durchschnittlichen $\mathrm{SaO}_{2}$ ist nicht nur unter RL, sondern auch unter $\mathrm{CF}$ und $\mathrm{D}$ hoch signifikant. Sie unterscheidet sich zwischen $\mathrm{O}_{2} \mathrm{CF}$ und $\mathrm{O}_{2}$ D nicht signifikant und ist auch unter RL nicht signifikant stärker ausgeprägt als unter den beiden anderen Applikationsformen (Tab. 2). Unter RL liegt jedoch erwartungsgemäß die durchschnittliche $\mathrm{SaO}_{2}$ vor Belastungsbeginn signifikant niedriger als unter CF bzw. D. Bei Sauerstoffzufuhr in den ersten 3 Minuten der Phase 1 steigt die $\mathrm{SaO}_{2}$ unter $\mathrm{CF}$ und D um den gleichen Betrag an (unter RL erwartungsgemäß keine nennenswerte Änderung) und erreicht in den nachfolgenden 3 Minuten nahezu ein Plateau. In den ersten 3 Minuten nach Belas-
Abb. 5 Änderung der $\mathrm{SaO} 2\left(\Delta \mathrm{SaO}_{2}\right)$ jeweils in den 1. und 2. 3 Minuten jeder Phase unter $2 \mathrm{I} \mathrm{O}_{2}$ kontinuierlichem Fluss (CF), $2 \mathrm{I} \mathrm{O}_{2}$ Demand (D) und unter Raumluft (RL).
Abb. 6 Verlauf der $\mathrm{SaO}_{2}$ in Phase 1, 2 und 3 eines COPD-Patienten mit schwerer respiratorischer Insuffizienz.

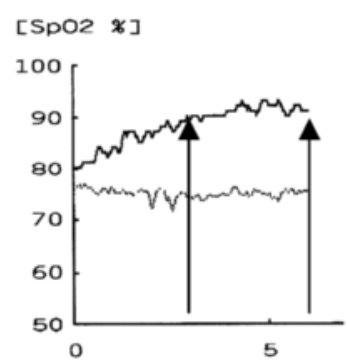

Phase 1: Vor Belastung

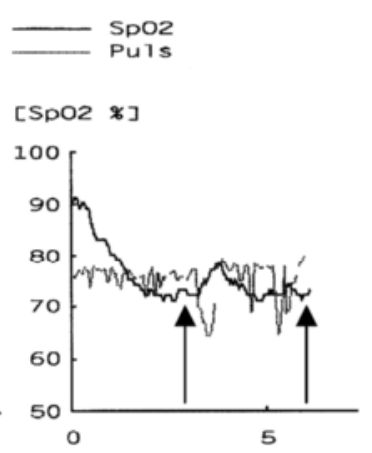

Phase 2: 6MWT

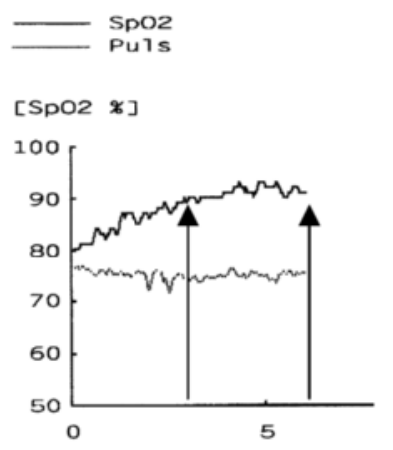

Phase 3: Nach Belastung 
Tab. 3 Einteilung in 2 Gruppen: Gruppe I (10 Pat.) mit kürzester, Gruppe II (10 Pat.) mit längster Gehstrecke im 6MWT unter 2 I O 2 CF: Durchschnittliche $\mathrm{SaO}_{2}$ vor und unter Belastung sowie Änderung der $\mathrm{SaO}_{2}(\Delta \mathrm{SaO})$ unter Belastung; $\Delta \mathrm{SaO}_{2}$ in den ersten 3 Minuten der jeweiligen Phase vor, unter und nach Belastung; $\triangle$ BORG: Änderung BORG nach/vor 6MWT; $\triangle 6 \mathrm{MWT}$ : Änderung Gehstrecke unter D (D-CF) und unter RL (RL-CF). Erklärung von CF, D und RL siehe Abb. 1

\begin{tabular}{|c|c|c|c|}
\hline Messwerte & Gruppe I & Gruppe II & Signifikanz $p$ \\
\hline $\mathrm{pO}_{2}$ & $54,7 \pm 8,0$ & $57,2 \pm 5,8$ & 0,36 \\
\hline FEV1 & $0,76 \pm 0,27$ & $1,26 \pm 0,56$ & 0,026 \\
\hline FEV1\% Soll & $35,6 \pm 17,1$ & $49,0 \pm 19,4$ & 0,11 \\
\hline \multicolumn{4}{|l|}{$\mathrm{O}_{2} \mathrm{CF}:$} \\
\hline $\begin{array}{l}\text { Gehstrecke 6MWT (m) } \\
\text { Gehstrecke \% Soll }\end{array}$ & $\begin{array}{c}198 \pm 34 \\
40,2 \pm 7,5\end{array}$ & $\begin{array}{c}350 \pm 22 \\
66,3 \pm 7,1\end{array}$ & $\begin{array}{l}0,0002 \\
0,0002\end{array}$ \\
\hline $\mathrm{SaO}_{2}$ vor Bel. (\%) & $93,4 \pm 2,8$ & $94,7 \pm 2,0$ & 0,24 \\
\hline $\mathrm{SaO}_{2}$ unter Bel. (\%) & $87,4 \pm 8,5$ & $90,2 \pm 3,0$ & 0,62 \\
\hline$\triangle \mathrm{SaO}_{2}$ unter Bel. (\%) & $-5,9$ & $-4,5$ & 0,97 \\
\hline $\begin{array}{l}\triangle \mathrm{SaO} 2 \\
\text { 1. } 3 \text { min vor Bel. (\%) } \\
\text { 1. } 3 \text { min unter Bel. (\%) } \\
\text { 1. } 3 \text { min nach Bel. (\%) }\end{array}$ & $\begin{array}{r}5,3 \pm 4,0 \\
-9,0 \pm 6,9 \\
6,9 \pm 8,1\end{array}$ & $\begin{array}{r}3,6 \pm 1,1 \\
-5,1 \pm 2,4 \\
3,1 \pm 2,1\end{array}$ & \\
\hline$\triangle B O R G$ unter Bel. & $3,4(3,1 \rightarrow 6,5)$ & $3,4(2,1 \rightarrow 5,5)$ & 0,97 \\
\hline \multicolumn{4}{|l|}{$\mathrm{O}_{2} \mathrm{D}$} \\
\hline Gehstrecke 6MWT (m) & $206 \pm 48$ & $379 \pm 28$ & $<0,0002$ \\
\hline$\triangle 6 M W T D-C F(m)$ & $8 \pm 22$ & $29 \pm 21$ & 0,08 \\
\hline $\mathrm{SaO}_{2}$ vor Bel. (\%) & $92,9 \pm 3,5$ & $94,2 \pm 1,4$ & 0,38 \\
\hline $\mathrm{SaO}_{2}$ unter Bel. (\%) & $87,9 \pm 5,7$ & $89,5 \pm 4,1$ & 0,68 \\
\hline$\triangle \mathrm{SaO}_{2}$ unter Bel. (\%) & $-4,9$ & $-4,7$ & 0,97 \\
\hline $\begin{array}{l}\Delta \mathrm{SaO}_{2} \\
\text { 1. } 3 \text { min vor Bel. (\%) } \\
\text { 1. } 3 \text { min unter Bel. (\%) } \\
\text { 1. } 3 \text { min nach Bel. (\%) }\end{array}$ & $\begin{array}{r}4,7 \pm 3,5 \\
-6,5 \pm 6,3 \\
6,0 \pm 5,7\end{array}$ & $\begin{array}{r}3,1 \pm 1,5 \\
-5,6 \pm 3,3 \\
3,2 \pm 2,9\end{array}$ & \\
\hline$\triangle B O R G$ unter Bel. & $3,0(3,4 \rightarrow 6,4)$ & $3,2(2,1 \rightarrow 5,3)$ & 1,0 \\
\hline \multicolumn{4}{|l|}{ RL: } \\
\hline Gehstrecke 6MWT (m) & $185 \pm 51$ & $390 \pm 54$ & 0,0002 \\
\hline$\triangle 6 M W T R L-C F(m)$ & $-13 \pm 45$ & $40 \pm 46$ & 0,02 \\
\hline $\mathrm{SaO}_{2}$ vor Bel. (\%) & $89,0 \pm 3,8$ & $91,7 \pm 1,2$ & 0,10 \\
\hline $\mathrm{SaO}_{2}$ unter Bel. (\%) & $81,5 \pm 6,4$ & $85,0 \pm 4,3$ & 0,14 \\
\hline$\triangle \mathrm{SaO}_{2}$ unter Bel. (\%) & $-7,6$ & $-6,7$ & 0,57 \\
\hline $\begin{array}{l}\triangle \mathrm{SaO}_{2} \\
\text { 1. } 3 \text { min vor Bel. (\%) } \\
\text { 1. } 3 \text { min unter Bel. (\%) } \\
\text { 1. } 3 \text { min nach Bel. (\%) }\end{array}$ & $\begin{array}{r}-0,4 \pm 1,2 \\
-8,3 \pm 6,3 \\
6,6 \pm 6,3\end{array}$ & $\begin{array}{r}-0,3 \pm 0,8 \\
-7,1 \pm 4,3 \\
6,3 \pm 5,5\end{array}$ & \\
\hline$\triangle B O R G$ unter Bel. & $3,3(3,3 \rightarrow 6,6)$ & $3,5(2,4 \rightarrow 5,9)$ & 0,97 \\
\hline
\end{tabular}

tungsbeginn tritt ein signifikanter Abfall der $\mathrm{SaO}_{2}$ ein, der in den letzten 3 Minuten der Belastungsphase nicht mehr wesentlich zunimmt. In den ersten 3 Minuten nach Belastungsende steigt die $\mathrm{SaO}_{2}$ wieder auf den Ausgangswert an, während sie in den letzten 3 Minuten der Ruhephase konstant bleibt (Abb.5). Ein Beispiel für den Verlauf der $\mathrm{SaO}_{2}$-Kurve in den jeweiligen 3 Phasen ist Abb. 6 zu entnehmen. Die Gehstrecke in den ersten $3 \mathrm{Mi}-$ nuten der Gesamtgehstrecke liegt unter CF, D bzw. RL zwischen 51,8 und 53,2\%; somit ist die Gehstrecke in den letzten 3 Minuten der Belastung trotz niedrigerer $\mathrm{SaO}_{2}$ nicht wesentlich kürzer. Das Atemnotempfinden unter Belastung ( $\triangle B O R G$ ) nimmt im Gesamtkollektiv bei Vergleich zwischen CF, D und RL gleich stark zu (Tab. 2). Auch der Vergleich zwischen Gruppe I und II ergibt keinen Unterschied. Lediglich das Atemnotempfinden vor Belas- tungsbeginn liegt in Gruppe I bei allen drei Applikationsformen um etwa einen Skalenpunkt höher als in Gruppe II (Tab. 3). Ähnliches gilt für das Pulsverhalten: vor Belastungsbeginn (Phase 1) ist die Herzfrequenz in Gruppe I höher als in Gruppe II; jedoch ist kein Unterschied in der Pulsänderung zwischen den drei Applikationsformen oder zwischen beiden Gruppen zu erkennen (Das Pulsverhalten wurde nicht dargestellt).

\section{Diskussion}

Unsere Untersuchungen zeigen, dass sich im Gesamtkollektiv die Gehstrecke im 6MWT bei einer Sauerstoff-Flussrate von 2 1/min mit kontinuierlichem Fluss (CF) und bei einem Demand-System 
(D), aber auch bei Raumluft (RL) nicht signifikant unterscheidet. Sie zeigen ferner, dass die Abnahme der Sauerstoffsättigung unter D im Vergleich zu CF nicht signifikant different ist, so dass beide Formen der Sauerstoff-Zufuhr bei dieser Flussrate gleich effizient sind. Nachdem unter Verwendung des Demand-Systems der Sauerstoffverbrauch etwa zwei [31] bis sieben Mal [32] geringer ist, ergibt sich hieraus ein erhebliches Einsparpotenzial. Die Mehrzahl der Patienten bevorzugt jedoch aus subjektiven Gründen (Strömungsgeräusch des Demand-Ventils) den kontinuierlichen Fluss (CF: 12, D: 8, indifferent: 7).

Bei nur geringer intraindividueller Änderung der Gehstrecke unter D im Vergleich zu CF besteht eine hoch signifikante Korrelation zwischen den Gehstrecken und damit hohe Reproduzierbarkeit des 6MWT. Die Zunahme der Gehstrecke von 6,2\% im Gesamtkollektiv ist überwiegend auf die Zunahme bei den Patienten mit langer Gehstrecke (Gruppe II) zurückzuführen. Dieser Trend setzt sich unter RL fort: Gruppe II kann die Gehstrecke auch unter RL weiter steigern, Gruppe I erfährt eine Abnahme. Die Zunahme der Gehstrecke in Gruppe II ist ein klarer Lerneffekt. Offen bleibt die Frage, inwieweit in dieser Gruppe bei der dritten Belastungsuntersuchung erneut Sauerstoff an Stelle von Raumluft zu einer weiteren Zunahme, somit zu einem noch größeren Lerneffekt geführt hätte. Beide Gruppen unterscheiden sich nur in den absoluten $\mathrm{FEV}_{1}$-Werten, nicht jedoch im Schweregrad der respiratorischen Insuffizienz, in der Abnahme der Sauerstoffsättigung unter Belastung und auch nicht in der $\mathrm{Zu}-$ nahme des Atemnotempfindens.

Die American Thoracic Society hat im Jahr 2002 Richtlinien für den 6MWT veröffentlicht [33]. Diese Richtlinien wurden bei unserer Untersuchung weitgehend berücksichtigt. Entgegen den Empfehlungen betrug die Länge des Korridors (mit $25 \mathrm{~m}$ ) weniger als $30 \mathrm{~m}$. Eine Multi-Center-Studie hat jedoch ergeben, dass eine Streckenlänge zwischen $50(15,2 \mathrm{~m})$ und $164(50,0 \mathrm{~m}) \mathrm{ft}(1$ $\mathrm{ft}=0,3048 \mathrm{~m}$ ) keinen signifikanten Einfluss auf die Gesamtgehstrecke hat [34]. Demgegenüber ist die Gehstrecke bei ovalem oder kreisförmigen Streckenverlauf um etwa $10 \%$ länger, da keine Wendung am Ende des geraden Gehweges erforderlich ist [35]. Sciurba u. Mitarb. wiesen in ihrer Multi-Center-Studie mit einem Kollektiv von 470 Patienten nach, dass Patienten mit längerer Gehstrecke im ersten Test zu einer größeren Zunahme tendierten, wenn der Test am folgenden Tag wiederholt wurde [35]. Wir kommen bei unseren Untersuchungen zum gleichen Ergebnis. Hien und Morr geben einen Lerneffekt bei einem $\mathrm{FEV}_{1}<30 \%$ Soll von 20-60 m, bei einem $\mathrm{FEV}_{1} 30-50 \%$ Soll von $25-75 \mathrm{~m}$ an [22]. Wir sahen zwar eine Abhängigkeit zwischen der Zunahme der Gehstrecke und dem absoluten $\mathrm{FEV}_{1}$, nicht jedoch dem $\mathrm{FEV}_{1} \%$ Soll. Nach einer Untersuchung von Gibbons war bei 79 gesunden Probanden die Gehstrecke bei wiederholter Belastung am gleichen Tag um durchschnittlich 6,6\% länger als beim ersten Versuch [36]. Bei Patienten mit chronischen kardiopulmonalen Erkrankungen liegt die Zunahme der Gehstrecke zwischen 10 und $30 \%$ [21,37]. Leach u. Mitarb. fanden eine Zunahme um 17\%, jedoch erst bei der dritten Untersuchung; bei den folgenden 6MWT war der Lerneffekt gering, dementsprechend die Reproduzierbarkeit hoch [38]. Reproduzierbarkeit und Lerneffekt stehen in inverser Beziehung zueinander. Die unterschiedlichen Angaben über den Lerneffekt im 6MWT sind mit auf die Heterogenität des Patientenkollektivs zurückzuführen: nach unseren Er- gebnissen zeigt sich eine Zunahme der Gehstrecke absolut und in Prozent des Ausgangswertes überwiegend bei den Patienten mit langer Ausgangsgehstrecke. Die unterschiedliche Änderung der Gehstrecke bei beiden Gruppen ergibt sich auch, wenn die Gehstrecke in \%Soll berechnet wird.

Die Änderung der Sauerstoffsättigung ist nicht linear. Sauerstoffzufuhr vor Belastungsbeginn führt unter CF und D innerhalb 3 Minuten zu einer Aufsättigung ohne Zunahme in den folgenden 3 Minuten. Unter Belastung tritt eine rasche Entsättigung ein. Gleichwohl ist die Gehstrecke in der zweiten Hälfte der Belastungszeit nur geringfügig kürzer und die Patienten setzen trotz niedriger Sättigung ihre Belastung kontinuierlich fort. Abbruch der Belastung führt binnen 3-10 Minuten zur Wiederaufsättigung. Trotz einer Sauerstoff-Flussrate von 2 1/min fällt unter Belastung bei 16 (unter CF) bzw. bei 14 (unter D) der 27 Patienten die Sättigung auf Werte unter $90 \%$ ab, so dass bei diesen Patienten die Flussrate mehr als $21 / \mathrm{min}$ betragen sollte. Mit dem von uns verwendeten portablen Sauerstoffgerät kann unter CF die maximale Flussrate von $21 /$ min nicht überschritten werden; mit dem Demand-System hingegen können Flussraten bis $51 / m i n$ angeboten werden. Überraschend ist, dass die Zunahme des Atemnotempfindens unter RL nicht signifikant stärker ausgeprägt ist als unter Sauerstoffgabe. Belastung führt bei Patienten mit COPD zu einer dynamischen Lungenüberblähung mit Abnahme der Inspiratorischen Kapazität (IC). Die Abnahme der IC ( $\Delta \mathrm{IC})$ korreliert signifikant mit der Zunahme der Atemnot $(\triangle B O R G)$ $[39,40]$. Bei stärkerer Limitierung der Belastbarkeit mit stärkerer Abnahme der IC in Gruppe I wäre auch ein stärkerer Anstieg des Atemnotempfindens zu erwarten gewesen. Unser Ergebnis widerspricht diesen Erwartungen.

In einer Doppelblind-Studie untersuchten Nandi u. Mitarb. 34 Patienten, denen über 10 Minuten vor bzw. über 5 Minuten nach einem 6MWT RL bzw. 28\% Sauerstoff appliziert wurden. Es ergab sich eine geringe, nicht signifikante Zunahme der Gehstrecke von $5 \mathrm{~m}$ in der Gruppe mit Sauerstoff. Bei Änderung des Atemnotempfindens und Erholungszeit bestanden keine Unterschiede [41]. Stevenson und Calverley verabreichten bei $18 \mathrm{~Pa}-$ tienten nach Ergometerbelastung Sauerstoff $\left(\mathrm{FIO}_{2} 0,4\right)$ bzw. Raumluft. Unter Sauerstoff war der Schweregrad der Überblähung rascher rückläufig; Unterschiede in der Rückbildung des Dypnoe-Empfindens ergaben sich jedoch nicht [42]. Die Ergebnisse von Nandi wurden somit bestätigt. Auch wir stellten unter Sauerstoff im Vergleich zu Raumluft keine stärkere Abnahme der Dyspnoe $(-\triangle B O R G)$ nach Belastungsende fest (Tab. 2). In einer weiteren Studie zeigten Jolly u. Mitarb., dass bei Patienten mit Abnahme der Sättigung um $\geq 5 \%$ und auf Werte $<90 \%$ im 6MWT unter RL ihre Gehstrecke unter Sauerstoffinsufflation um $22 \%$ zunahm, während dies bei Patienten ohne Abnahme nicht der Fall war. In beiden Gruppen nahm jedoch unter Sauerstoffzufuhr das Atemnotempfinden ab [11]. Ein Vergleich dieser Beobachtungen mit unseren Ergebnissen ist wegen des unterschiedlichen Patientenkollektivs und Versuchsablaufs nur begrenzt möglich. Der Ruhe- $\mathrm{pO}_{2}$ lag in dem Patientengut von Jolly bei über 70 (in unserem Kollektiv bei 55) Torr. Bei Abnahme der Sättigung unter Belastung wurde Sauerstoff bis zu 12 1/min appliziert. Die Sauerstoff-Flussrate wurde bei unserer Untersuchung hingegen mit $21 /$ min konstant gehalten. 
Wir sehen in der Verwendung des Demand-Flüssig-Sauerstoffsystems bei einer Flussrate von 2 1/min keinen Nachteil in Bezug auf das Verhalten der Sauerstoffsättigung. Senn u. Mitarb. verglichen bei 12 Patienten mit COPD unter Belastung ein DemandSystem (Puritan-Bennett Companion Oxygen Saver; COS) mit kontinuierlichem Fluss bei unterschiedlichen Sauerstoff-Flussraten und fanden eine enge Korrelation zwischen beiden Systemen in Bezug auf den $\mathrm{pO}_{2}$ selbst bei hoher Atemfrequenz; bei Anwendung der Lippenbremse lag der $\mathrm{pO}_{2}$ unter kontinuierlichem Fluss höher [43]. Das gleiche System wurde von Bower u. Mitarb. verglichen: bei einer mit 6 COPD-Patienten sehr kleinen Gruppe ergab sich unter Ergometriebelastung mit einer Sauerstoff-Flussrate von $21 /$ min in Bezug auf die durchschnittliche Sättigung kein signifikanter Unterschied. Der Sauerstoff-Verbrauch lag bei Verwendung des Demand-Systems bei $44 \%$ im Vergleich zu kontinuierlichem Fluss [31]. Andere Arbeitsgruppen sehen beide Applikationsformen nicht als gleichwertig an. Roberts u. Mitarb. kamen zu dem Ergebnis, dass im 6MWT unter einer SauerstoffFlussrate von $21 /$ min die Zeitdauer der Entsättigung unter 90\% unter D signifikant länger war als unter CF [44]. Die niedrigste Sauerstoffsättigung wurde unter dem Demand-System festgestellt (bei unserer Untersuchung fand sich die niedrigste Sättigung unter CF). Übereinstimmend mit unseren Ergebnissen jedoch war die Zunahme des Atemnotempfindens, hier durch die Änderung in der Visuellen Analog-Skala (VAS) ausgedrückt, unter CF, D und RL nicht unterschiedlich. Im Unterschied zu unserem Versuchsablauf war bei Roberts die Reihenfolge der Belastungsbedingungen randomisiert. Hagarty wies bei 15 Patienten mit COPD unter Belastung mit dem Demand-Ventil eine niedrigere Sättigung im Vergleich zu kontinuierlicher Sauerstoff-Zufuhr nach. Als Belastungsformen wurden Alltagstätigkeiten wie An- und Auskleiden oder Arbeiten mit den Armen gewählt [45]. Eventuell führen unterschiedliche Belastungsformen auch zu unterschiedlichen Ergebnissen.

Bei unserer Untersuchung war die Reihenfolge der Applikationsformen nicht randomisiert. Damit war der Unterschied durch den Lerneffekt überlagert; die Aussagekraft der Ergebnisse ist hierdurch eingeschränkt. Hierin liegt eine Schwäche unserer Untersuchung. Bei nahezu identischem Verhalten der Sauerstoffsättigung unter $C F$ und $D$ nehmen wir jedoch an, dass die Reihenfolge bei den beiden Sauerstoff-Applikationsformen ohne signifikanten Einfluss auf das Ergebnis gewesen wäre. Unter der Voraussetzung, dass $\mathrm{CF}$ und $\mathrm{D}$ bei $\mathrm{O}_{2} 2 \mathrm{l} / \mathrm{min}$ gleiche Untersuchungsbedingungen bieten, kann eine Aussage über den Lerneffekt getroffen werden. Auch konnte bei der von uns festgelegten Reihenfolge dargestellt werden, dass selbst unter RL ein weiterer Lerneffekt möglich ist, dies jedoch nur in der Gruppe mit längerer Gehstrecke.

Das von uns verwendete Sauerstoffsystem lässt keine höheren Flussraten als 2 1/min kontinuierlichen Fluss zu. Diesem Nachteil ist das vergleichsweise geringe Gewicht von 1,95 kg entgegen zu stellen. Sauerstoffsysteme mit höherem Gewicht finden bei Patienten mit Osteoporose und Muskelatrophie keine Akzeptanz, insbesondere, wenn die Belastungsdauer über sechs Minuten hinausgeht. So beschreibt Leach, dass allein das Füllgewicht von $3,5 \mathrm{~kg}$ des von ihm verwendeten Sauerstoffsystems die Gehstrecke um $14,1 \%$ reduziert [38].
Inwieweit belastungsinduzierte Hypoxämien bei einem Ruhe$\mathrm{pO}_{2}$ von $>55$ Torr Lebensqualität und/oder Prognose verschlechtern bzw. ob Sauerstoffgabe allein unter Belastung einen Langzeit-Vorteil ergibt, ist nicht sicher bekannt [6,11]. Zwei Untersuchungen ergaben eine direkte Korrelation zwischen Abnahme des $\mathrm{pO}_{2}$ unter Belastung und dem Pulmonalarteriendruck in Ruhe und eine inverse Korrelation mit der Prognose [46,47].

Die Belastbarkeit von Patienten mit COPD ist nicht allein durch ventilatorische Einschränkungen limitiert. So zeigte eine Untersuchung von Celli u. Mitarb. an zehn Patienten mit COPD, dass im Vergleich zu Gesunden Herzminutenvolumen und Herzindex unter Belastung signifikant geringer anstiegen [48]. Könnte somit eine auf eine günstige Beeinflussung dieser kardialen Parameter ausgerichtete Therapie auch die Belastbarkeit beeinflussen?

Aktuell sind Studien zu folgenden offenen Fragen von Interesse: Welche Medikamente in welcher Dosierung haben Einfluss auf die Gehstrecke? In welchem Maße unterliegt der 6MWT infolge der unterschiedlichen Wirkdauer der Medikation auch einer tageszeitlichen Rhythmik? Wie wirkt sich eine (wie lange) Ruhephase vor Belastung auf die Gehstrecke aus? Über welchen Zeitraum zwischen zwei Untersuchungen ist der 6MWT reproduzierbar bzw. bleibt der Lerneffekt erhalten?

Zusammenfassend sind Gehstrecke und Verhalten der Sauerstoffsättigung unter einer Sauerstoff-Flussrate von $21 /$ min unter kontinuierlichem Fluss und unter Verwendung eines DemandSystems vergleichbar. Das Demand-System ermöglicht daher einen realen Spareffekt. Eine Flussrate von 2 1/min reicht bei mehr als der Hälfte unserer Patienten nicht aus, um einen Abfall der Sauerstoffsättigung auf Werte unter $90 \%$ zu vermeiden. Patienten mit größerem $\mathrm{FEV}_{1}$ können unabhängig vom Schweregrad der respiratorischen Insuffizienz ihre Gehstrecke bei Wiederholung auch unter Raumluft steigern. Die Gehstrecke pro Zeiteinheit ist während des 6MWT nahezu konstant, so dass nach $3 \mathrm{Mi}-$ nuten die Gesamtgehstrecke vorausgesagt werden kann. Bei nahezu gleicher Gehstrecke in der zweiten Hälfte des 6MWT ist in dieser Zeitphase die Sauerstoffsättigung jedoch deutlich niedriger als in der ersten Hälfte.

Der Sechs-Minuten-Gehtest ist ein bewährtes Instrument zur Beurteilung der Belastbarkeit bei Patienten mit COPD. Wir erkennen, dass bereits Alltagsbelastung trotz Sauerstoffzufuhr zu einem teilweise dramatischen Abfall der Sauerstoffsättigung führen kann, der nach Belastungsende jedoch nur kurz anhält. 


\section{Literatur}

${ }^{1}$ McSweeny AJ, Grant I, Heaton RK et al. Life quality of patients with chronic obstructive pulmonary disease. Arch Intern Med 1982; 142: $473-478$

2 Okubadejo AA, Paul EA, Jones PW et al. Quality of life in patients with chronic obstructive pulmonary disease and severe hypoxemia. Thorax 1996; $51: 44-47$

${ }^{3}$ Burrows D, Earle RH. Course and prognosis of chronic obstructive lung disease: a prospective study in 200 patients. N Engl J Med 1969; 280: $397-404$

${ }^{4}$ Nocturnal Oxygen Therapy Trial Group. Continuous or nocturnal oxygen therapy in hypoxic chronic obstructive lung disease. Ann Intern Med 1980; 93: 391 - 398

${ }^{5}$ Medical Research Council Working Party. Long term domiciliary oxygen therapy in chronic hypoxic cor pulmonale complicating chronic bronchitis and emphysema. Lancet 1981; 1: 681-686

${ }^{6}$ Deutsche Gesellschaft für Pneumologie. Wissenschaftliche Sektion: Klinische Pneumologie. Leitlinien zur Langzeit-Sauerstofftherapie. Pneumologie 2001; 55: 454-464

${ }^{7}$ Ries AL, Farrow JT, Clausen JL. Pulmonary function tests cannot predict exercise-induced hypoxemia in chronic obstructive pulmonary disease. Chest 1988; 93: 454-459

${ }^{8}$ Freitag AP, Summers E, Hamilton AL et al. Behavior of arterial oxygen saturation during exercise in normal subjects and patients with pulmonary and cardiac impairment. Chest 1995; 108: 140S-000

${ }^{9}$ Eaton T, Garrett JE, Young P et al. Ambulatory oxygen improves quality of life of COPD patients: a randomised controlled study. Eur Respir J 2002; 20: 306-312

${ }^{10}$ Woodcock AA, Gross ER, Geddes DM. Oxygen relieves breathlessness in "pink puffers". Lancet 1981; 1: 907-909

${ }^{11}$ Jolly EC, Di Boscio V, Aguirre L et al. Effects off supplemental oxygen during activity in patients with advanced COPD without severe resting hypoxemia. Chest 2001; 120: 437-443

${ }^{12}$ Dean NC, Brown JK, Himelman RB et al. Oxygen may improve dyspnoea and endurance in patients with chronic obstructive pulmonary disease and only mild hypoxemia. Am Rev Respir Dis 1992; 146: $941-945$

${ }^{13}$ Bradley BL, Garner AE, Billiu D et al. Oxygen-assisted exercise in chronic obstructive lung disease: the effect on exercise capacity and arterial blood gas tensions. Am Rev Respir Dis 1978; 118: 239-243

${ }^{14}$ Emtner M, Porszasz J, Burns M et al. Benefits of supplemental oxygen in exercise training in nonhypoxemic chronic obstructive pulmonary disease patients. Am J Respir Crit Care Med 2003; 168: 1034- 1042

15 Rooyackers JM, Dekhuijzen PN, Herwaarden CLVan et al. Training with supplemental oxygen in patients with COPD and hypoxaemia at peak exercise. Eur Respir J 1997; 10: 1278 - 1284

${ }^{16}$ Dewan NA, Bell W. Effect of low flow and high flow oxygen delivery on exercise tolerance and sensation of dyspnea. Chest 1994; 105: $1061-1065$

17 O'Donnell DE, Bain DJ, Webb KA. Factors contributing to relief of exertional breathlessness during hyperoxia in chronic airflow limitation. Am J Respir Crit Care Med 1997; 155: 530-535

18 Chatila W, Nugent T, Vance G et al. The effects of high-flow vs. lowflow oxygen on exercise in advanced obstructive airways disease. Chest 2004; 126: $1108-1115$

${ }^{19}$ Somfay A, Porszasz J, Lee SM et al. Dose-response effect of oxygen on hyperinflation and exercise endurance in nonhypoxaemic COPD patients. Eur Respir J 2001; 18: 77-84

20 Solway S, Brooks D, Lacasse $Y$ et al. A qualitative systematic overview of the measurement properties of functional walks used in the cardiorespiratory domain. Chest 2001; 119: $256-270$

${ }^{21}$ Guyatt GH, Prugsley SO, Sullivan MJ et al. Effect of encouragement on walking test performance. Thorax 1984; 39: 818-822

22 Hien P, Morr H. 6-Minuten-Gehtest in der pneumologischen und kardiologischen Diagnostik. Pneumologie 2002; 56: 558-566

${ }^{23}$ Enright PL, McBurnie MA, Bittner V et al. The 6-min walk test. Chest 2003; 123: 387-398

${ }^{24}$ Bowen JB, Vott JJ, Thrall RS et al. Functional status and survival following pulmonary rehabilitation. Chest 2000; 118: $697-703$
${ }^{25}$ Enright PL, Sherrill DL. Reference equations for the six-minute walk in healthy adults. Am J Respir Crit Care Med 1998; 158: 1384-1387

${ }^{26}$ Troosters T, Gosselink R, Decramer M. Six minute walking distance in healthy elderly subjects. Eur Respir J 1999; 14: 270-274

${ }^{27}$ Gerardi DA, Lovett L, Benoit-Connors ML et al. Variables related to increased mortality following out-patient pulmonary rehabilitation. Eur Respir J 1996; 9: 431 - 435

28 Pinto-Plata VM, Cote C, Cabral $\mathrm{H}$ et al. The 6-min walk distance: change over time and value as a predictor of survival in severe COPD. Eur Respir J 2004; 23: 28-33

${ }^{29}$ Borg GA. Psychophysical bases of perceived exertion. Med Sci Sports Exerc 1982; 14: 377-381

${ }^{30}$ McGavin CR, Artvinli M, Naoe $\mathrm{H}$ et al. Dyspnoea, disability, and distance walked: comparison of estimates of exercise performance in respiratory rates. Br Med J 1978; 2: 241 - 243

${ }^{31}$ Bower JS, Brook CJ, Zimmer K et al. Performance of a demand oxygen saver system during rest, exercise, and sleep in hypoxemic patients. Chest 1988; 94: 77-80

32 Tiep BL, Carter R, Brooke $\mathrm{N}$ et al. Demand oxygen delivery during exercise. Chest 1987; 91: 15-20

${ }^{33}$ American Thoracic Society Statement. Guidelines for the six-minute walk test. Am J Respir Crit Care Med 2002; 166: 111 - 117

${ }^{34}$ Weiss RA et al. Six minute walk test in severe COPD: reliability and effect of walking course layout and length. San Francisco: Paper presented at ACCP Conference, September 2002

35 Sciurba F, Criner GJ, Lee SM et al. Six minute walk distance in chronic obstructive pulmonary disease. Reproducibility and effect of walking course layout and length. Am J Respir Crit Care Med 2003; 167: $1522-1527$

${ }^{36}$ Gibbons WJ, Fruchter N, Sloan S et al. Reference values for a multiple repetition 6-minute walk test in healthy adults older than 20 years. J Cardiopulm Rehabil 2001; 21:87-93

${ }^{37}$ Knox AJ, Morrison JF, Muers MF. Reproducibility of walking test results in chronic obstructive pulmonary disease. Thorax 1988; 43: 388-392

38 Leach RM, Davidson AC, Chinn S et al. Portable liquid oxygen and exercise ability in severe respiratory disability. Thorax 1992; 47: 781 - 789

39 O'Donnell DE, Lam M, Webb KA. Measurement of symptoms, lung hyperinflation and endurance during exercise in chronic obstructive pulmonary disease. Am J Respir Crit Care Med 1998; 158: 1557 - 1565

${ }^{40}$ Marin JM, Carrizo SJ, Gascon M et al. Inspiratory capacity, dynamic hyperinflation, breathlessness and exercise performance during the 6-minute-walk test in chronic obstructive pulmonary disease. Am J Respir Crit Care Med 2001; 163: 1395 - 1399

${ }^{41}$ Nandi K, Smith AA, Crawford A et al. Oxygen supplementation before or after submaximal exercise in patients with chronic obstructive pulmonary disease. Thorax 2003; 58: 670-673

42 Stevenson NJ, Calverley PM. Effect of oxygen on recovery from maximal exercise in patients with chronic obstructive pulmonary disease. Thorax 2004; 59: 668-672

${ }^{43}$ Senn S, Wanger J, Fernandez E et al. Efficacy of a pulsed oxygen delivery device during exercise in patients with chronic respiratory disease. Chest 1989; 96: 467-472

${ }^{44}$ Roberts CM, Bell J, Wedzicha JA. Comparison of the efficacy of a demand oxygen delivery system with continuous low flow oxygen in subjects with stable COPD and severe oxygen desaturation on walking. Thorax 1996; 51: $831-834$

${ }^{45}$ Hagarty EM, Morton S, Skorodin MS et al. Comparison of three oxygen delivery systems during exercise in hypoxemic patients with chronic obstructive pulmonary disease. Am J Respir Crit Care Med 1997; 155: $893-898$

${ }^{46}$ Kawakami Y, Terai T, Yamamoto $\mathrm{H}$ et al. Exercise and oxygen inhalation in relation to prognosis of chronic obstructive pulmonary disease. Chest 1982; 81: $182-188$

${ }^{47}$ Keller CA, Shepard JW, Chun DS et al. Pulmonary hypertension in chronic obstructive pulmonary disease: multivariate analysis. Chest 1986; 90: $185-192$

48 Celli BR, Hoskere G, Mehta JB et al. Hemodynamic changes to exercise in patients with COPD and normal controls. Chest 2004; 126: 922S (Poster presentation) 